\title{
Internal Fixation of the Intra Articular Fractures of Calcaneum without Bone Grafting
}

\author{
G.A. Rajmohan ${ }^{1}, \mathrm{E} \mathrm{Siva}^{1}, \mathrm{~K}$. Thennarasu ${ }^{2}$ \\ ${ }^{1}$ (Department of Orthopedics, Thanjavur Medical College Hospital, Thanjavur, India) \\ ${ }_{2}^{2}$ (Department of Biostatistics, NIMHANS, Bengaluru, India)
}

\begin{abstract}
The management of calcaneal fractures range from closed treatment to open reduction internal fixation with different fixation. The radiological and functional outcome of 15 closed intra-articular Sander's type II and III calcaneal fractures fixed with locking calcaneal plate by extensile lateral approach was done. Properly selected cases with proper timing of surgery accepted results in both clinically and radiologically are achieved with few complications.
\end{abstract}

Keywords: Calcaneum Fractures, locking plate, internal fixation, Sander's

\section{Introduction}

With calcaneal fractures accounting for $65 \%$ of tarsal injuries and (70\%) of them are intra articular, internal fixation of the calcaneum as a method of treatment has attracted interest in recent times.(1,2) Most of these persons are manual workers and sole earning members of the family. The method to manage calcaneal fractures is unsettled.

Cotton and Wilson (1908) advocated for intra-articular fractures of calcaneum, a careful neglect by conservative management. With poor results from conservation, open reduction and internal fixation was advocated by Bohler (1931), Palmer 1948, Essex Lopresti (1952). Later complications of internal fixation occurred mainly due to non-availability of good fixation devices and effective anesthesia, good quality intraoperative radiography. Thus McLaughlin (1959) even compared open reduction and fixation of a calcaneal fracture to "nailing of a custard pie to the wall". So only later, between 1960s and70s most workers advocated of conservative management. It is observed that maintenance of Bohler's angle is necessary for satisfactory results along with maintenance of articular congruence of posterior facet of calcaneum and crucial angle of Gissane.(3) As an alternative to address the incongruity of joint surfaces in both acute and united calcaneal fractures Conn, Gallie, Harris, Dick advocated arthrodesis as the treatment of choice.

In the last 20 years, because of improved anesthesia, introduction of antibiotics, proper internal fixation devices, preoperative imaging CT and intra operative imaging surgeons were able to do operative fixation for many intra-articular fracture, obtaining good results.

Recent employment of locking compression plate for open reduction and internal fixation in displaced intraarticular fracture calcaneum produced good outcome. Results are more favourable in less comminuted as compared to more comminuted. (10)

The study have used locking compression plate in calacaneal fracture without using bone graft or bone cement for calcaneal fixation (6-9) Zhongguo et all,(11) however Leung et al(8), Thordarson et al(9), and Schildhauer et al(10) recommended use of bone graft or injectable bone cement to increase stability and compressive strength of fixation and quicker rehabilitation.

Objective of the study was to find the effectiveness of locking compression plate in maintaining reduction and restoring crucial angles in intra-articular fractures of calcaneum without bone grafting.

\section{Materials and Methods}

This prospective study was conducted in a trauma in patient setting in Government Thanjavur Medical College Hospital from 2013 to 2015 after due clearance from ethical committee. Patients with displaced closed intra articular fractures of calcaneum were selected and treated with open reduction and internal fixation with locking compression plate.

Open fractures, undisplaced calcaneal fractures, were excluded. Also patients with diabetic neuropathy with more chances of skin necrosis also were excluded. Patients with significant coexisting diseases contradicting any anaesthesia were excluded. There were seventeen fractures in fifteen cases. All cases were adult males in the age group of 21-60 years. Most (13) of these cases resulted from fall from height. Two had history of road traffic accident. All these cases presented with swelling and pain of the heel and inability to walk. They were evaluated with axial, lateral and anteroposterior radiographic views along with CT scan of the calcnaeum. T 
he fractures were classified according to Sander's classification; six fractures were of type 2 and nine were of type 3 classify the fractures. Their limbs were elevated and ice fomentation was applied and later crepe bandage was applied to reduce the edema. Most of the patients were operated within 10-14 days of injury. All the patients were informed the details about the surgery and consent was obtained.

\subsection{The Surgical Technique}

All the patients were administered spinal anaesthesia and general anaesthesia in one patient with spine injury. Patients were placed in lateral position, intra venous prophylatic antibiotics was administered and pneumatic tourniquet was applied later.

Through lateral extensile incision sural nerve was explored and protected within the retracted skin flap. Sub-periosteal dissection of anterior skin flap was done and retracted with $\mathrm{K}$ wire. Peroneal tendon sheath was dislocated over fibula.

One K-wire each was is passed over fibula, talar neck and cuboid. The lateral wall of calcaneum was excised to facilitate the mobility and reduction of the body and the tuberosity of calcaneum. Intra operative radiographic evaluation with image intensifier with lateral, axial and Antero posterior view was done and correction of varus, loss of height and increased width was done. This was temporarily stabilized (tuberosity to medial fragment) with $\mathrm{K}$ wires. Later reconstruction and fixation of intra articular fragment was done with $\mathrm{K}$ wires. Then insertion of 2 parallel partially threaded $4 \mathrm{~mm}$ cancellous screws was done. Reduction of anterior and middle facets and calcaneocuboid joint is done if necessary. The locking compression calcaneal plate with locking screws was used for fixation (Fig. 1). We used AO calcaneal locking plate (SSEPL ${ }^{\circledR}$ ) with 15 locking holes allowing for fixation of different fracture pattern. With special sleeve benders, superior and inferior arms of the plate can be molded to provide support for anterior processes and plantar fragments. Angled and ascending hole supports the sustentaculum and provides a better support to the calcaneo-talar articular surface. Multiple locking screws or standard small fragment screws provide unicortical and/or bi-cortical fixation. The threaded locking holes provide a fixed angle construct to support the articular surfaces of the calcaneus and permit multiple point of fixation to buttress small fragments. The locking hole provides 5 degree angulation when using $3.5 \mathrm{~mm}$ cortex screws and 15 degree angulation when using $2.7 \mathrm{~mm}$ cortex screws.
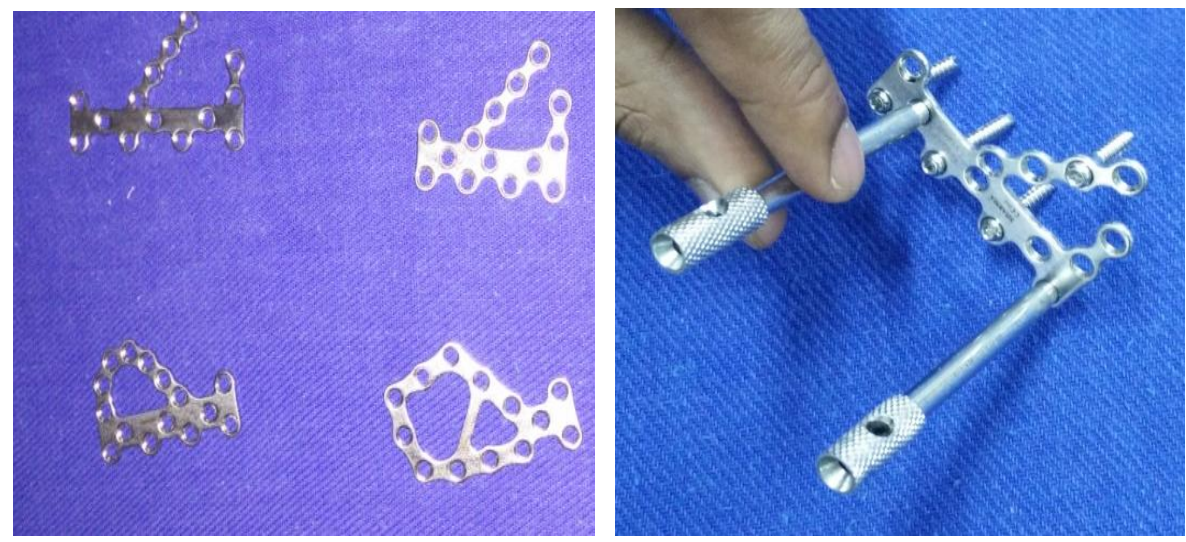

Fig 1. Various calcaneum locking plates

Corner stitch is done with 4-0 prolene and subcutaneous layer closure done with 2-0 Vicryl minimal sutures. Later rest of the wound is closed with staples or Ethilon. Sterile dressing followed by well-padded short below knee slab is applied.

Post operatively limb was kept elevated and intravenous antibiotics were given for five days. Closed suction drainage is kept for $24-48$ hours. The short leg splint is removed at fifth postoperative day. If the flap showed uncomplicated healing and the wound was healthy, early active range of motion was begun at that time. At second postoperative week, active range of motion of the ankle joint was started and gentle passive movement of subtalar joint was done. Suture removal was done at three weeks. Weight bearing is allowed after 12 weeks, till that time removable posterior splint should protect the limb. The plate removal is necessary if the patient shows symptoms that too after one year. There was one patient with associated fracture tibia and another patient with thoracolumbar fractures. The tibia fracture was treated with closed nailing in the same sitting. The thoracolumbar injury was treated conservatively and was administered general anesthesia for calcaneal fixation. The patient was on follow-up for a period of one year. The outcomes for this study was measured using the AOFAS score (American Orthopaedic Foot \& Ankle Hindfoot Score). This scoring system is extensively used and permits for comparison of results among several studies 
The descriptive statistics were expressed using frequency, percentages for categorical variables and mean, median along with standard deviation (SD) and range for continuous variables. After testing for normal distribution, comparison of pre and post-operative Bohler's and Gissane angle were carried out using paired sample $t$ test. $\mathrm{P}<0.05$ was considered statistically significant.

\section{Results}

All the 15 cases (17 fractures) are available for follow-up. The results of the surgical procedure analyzed radiographically (Fig. 2) and clinically with AOFAS score at 6 and 12 weeks post operatively and periodically once in every 6 weeks.

Bony union occurred in all patients. Two patients $(13.3 \%)$ had superficial wound infection which settled with appropriate antibiotics. Two patients $(13.3 \%)$ had superficial wound dehiscence among that one healed by secondary intention, other patient went for implant removal after radiological evidence of fracture union. Table 1 show that the change in pre and post-operative Bohler's and Gissane angles were significantly improved $(\mathrm{P}<0.001)$

Table 1: Descriptive statistics of preoperative and postoperative Bohler's and Gissane angles.

\begin{tabular}{|c|c|c|c|c|}
\hline & Pre-Operative & Post-Operative & Difference & P Value \\
\hline Bohler's Angle & $17.4 \pm 7.9$ & $28.8 \pm 6.6$ & $11.3 \pm 6.7$ & $<0.001$ \\
\hline Gissane Angle & $159.9 \pm 7.6$ & $140.9 \pm 10.1$ & $18.9 \pm 8.2$ & $<0.001$ \\
\hline
\end{tabular}
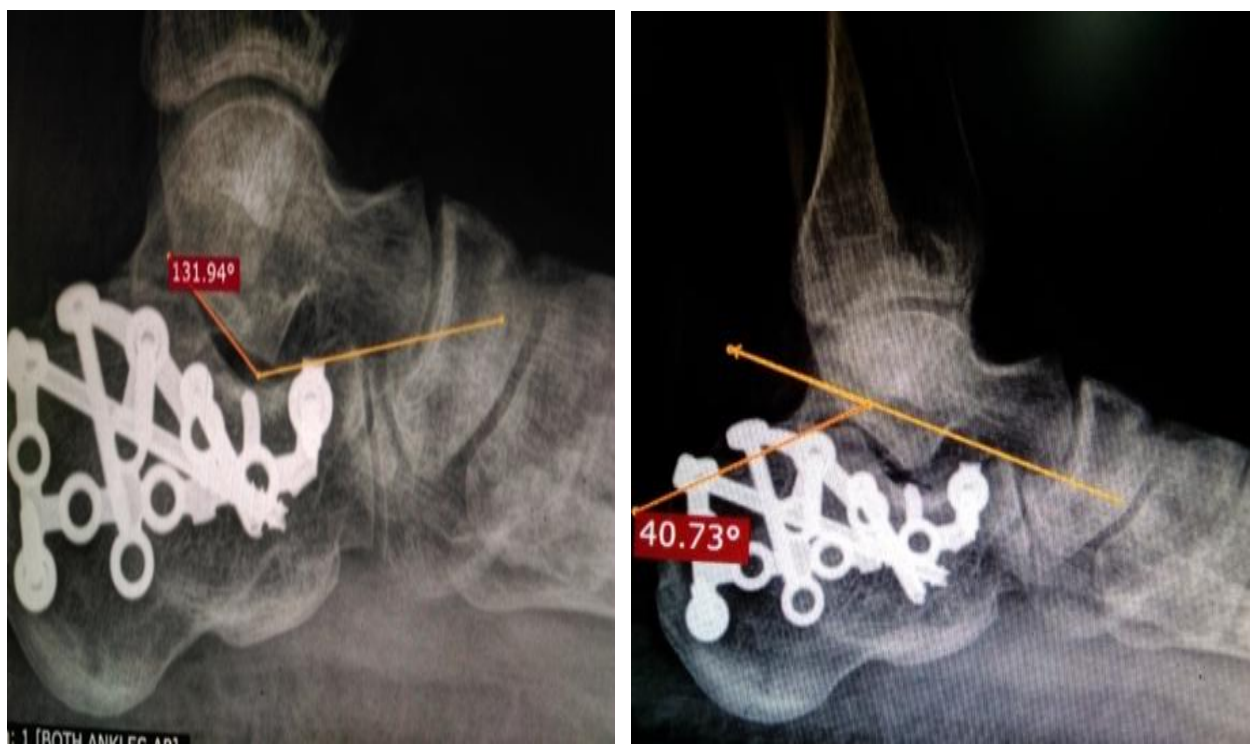

Fig 2.Preop radiograph and CT Sander's type 2 and postop radiographs showing the reconstruction of Gissane's and Bholer's angle

None of the patients $(0 / 15)$ had compartment syndrome, heel pad problems, peroneal tendinitis and a reflex sympathetic dystrophy

In our study the results as per AOFAS score were good in ten cases (66.7\%), fair in four cases $(26.7 \%)$ and poor in one case $(6.6 \%)$. Table 2 provide detailed descriptive statistics of each component of AOFAS score. The mean of total AOFAS score was $77.5 \pm 11.1$. There was superficial wound infection in two cases $(13.3 \%)$, minor wound dehiscence in two cases. Two of the cases $(13.3 \%)$, showed inadequate reduction with one went on to develop arthritis and sural nerve hypoaesthesia was noted in two cases.

Table 2: Descriptive statistics of all the cases with AOFAS

\begin{tabular}{|c|c|c|}
\hline & Mean \pm SD & Median(Range) \\
\hline Pain Score (40) & $28.2 \pm 8.8$ & $30(0-40)$ \\
\hline \multicolumn{3}{|l|}{ Function score (50) } \\
\hline Limitation; & $5.8 \pm 1.5$ & $7.0(4-7)$ \\
\hline Walking Distance & $3.9 \pm 0.8$ & $4.0(2-5)$ \\
\hline Walking Surface & $3.6 \pm 0.9$ & $3.0(3-5)$ \\
\hline Gait; & $7.3 \pm 1.6$ & $8.0(4-8)$ \\
\hline Sagittal motion; & $7.3 \pm 1.6$ & $8.0(4-8)$ \\
\hline HindFoot Motion & $3.4 \pm 1.0$ & $3.0(3-6)$ \\
\hline
\end{tabular}




\begin{tabular}{|l|c|c|}
\hline \multicolumn{1}{|c|}{ Ankle HindFoot Stability } & $8 \pm 0.0$ & $8.0(8-8)$ \\
\hline Alignment (10) & $10 \pm 0.0$ & $10(10-10)$ \\
\hline Total AOFAS score & $77.5 \pm 11.1$ & $7848-97)$ \\
\hline
\end{tabular}

\section{Discussion}

Seventeen calcaneum fractures in 15 patients were operated during follow-up they were assessed using AOFAS at $6^{\text {th }}$ and $12^{\text {th }}$ week. The outcome of this study based on AOFAS score were presented. This study outcome of $93 \%$ good or fair outcomes comparable with various studies done globally McReynolds ${ }^{(17)}$, Palmer $^{(19)}$ / Stephenson ${ }^{(16)} /$ Benirschke $^{(4)} /$ Thordarson. ${ }^{(9)}$ In the initial cases, we had complications like superficial wound infection, wound dehiscence and inadequate anatomical reduction. These complications developed due to delay in operative treatment. The poor results could be due to prolonged operative time in reducing the fractures which were taken up late for surgery. This is because fracture begins to consolidate. Later in our cases there were no skin complications.

Due to the risk of early complications, a clear idea about indications and contraindications and the timing of surgery are important. Pre and postoperative CT scans are essential.

Sustentaculum tali screw fixation was applied in two of our cases has provide the advantages of high stability, less postoperative pain, rapid functional recovery in treating Sanders type II and III calcaneal fractures as recommended ${ }^{(8)}$. The calcaneus locking plates address all the problem of calcaneal fracture fixation. with lower profile, better hold in bone, and versatile screw placement, reducing the need for graft or bone substitutes and allowing for earlier weight bearing. ${ }^{(13-14)}$ Thus at present the locking plates for comminuted calcaneus fractures is an acceptable method ${ }^{(15)}$. The above method not only restored anatomical height, width of calcaneum, but also its Bohler's and Gissiane's angles. This allows early mobilization. But surgical expertise and cost of the plate are two main limiting factors. The timing of the surgery is a vital and is decided by appearance of wrinkle sign. Those cases which were taken up for fixation early i.e less than 10 days had good results than those which were operated later.

\section{Conclusion}

The present study show that intra articular calcaneal fractures treated with open reduction and internal fixation with less traumatic techniques using stronger but malleable implants decrease the need for bone graft. This allow early weight bearing and also provides rigidity especially in osteoporotic cancellous bone. One of the limitations of the study that the sample size is small and without a comparable group for control.

\section{Acknowledgements}

We acknowledge Dr. S. Kumaravel, Professor of Orthopedics, Thanjavur Medical College Hospital, Thanjavur, India, for support during study and suggestion to improve the manuscript.

\section{References}

[1]. R.W. Sanders, M.P. Clare, Fractures of the calcaneus, Charles, James(Ed.,), Rockwood \& Green's fractures in Adults, ) $8^{\text {th }}$ ed. (Lippincott: Wolters Kluwer, 2015) 2639-2685(61).

[2]. S.W. Ishwaka, Fractures and dislocations of the foot, in S.T.Canale(Ed.,). Campbell's Operative Orthopaedics $12^{\text {th }}$ ed. (Philadelphia: Elsevier 2013) 4139-4157(88).

[3]. Sanders, Roy, Displaced intra articular fractures of calcaneum JBJS 82-A, No.2, Feb. 2000

[4]. D.P. Barei, C.Bellabarba, B.J. Sangeorzan, K. Benirschke. Fractures of the calcaneus. Orthop. Clin. North Am. 2002 Jan 33(1) : 263-85.

[5]. E.J. Harvey, L. Grujic, J.S. Early, K. Benirschke, B.J. Sangeorzan. Morbidity associated with ORIF of intra-articular calcaneus fractures using a lateral approach. Foot Ankle Tnt. 2001 Nov. 22(11) 868-73.

[6]. A. Pendse, R.N. Daveshwar, J. Bhatt, et al. Outcome after open reduction internal fixation of intra-articular fracture of calcaneum without use of bone graft. Indian J Orthop 2006;40(2):111-4.

[7]. D. Longino, R.E. Buckley. Bone graft in operative treatment of displaced intraarticular calcaneus fractures. Is it helpful? J Orthop Trauma 2001;15(4):280-6.

[8]. K.S. Leung, W.S. Chan, W.Y. Shen, et al. Operative treatment of intraarticular fractures of the os calcis - the role of rigid internal fixation and primary bone grafting: preliminary results. J Orthop Trauma 1989;3(3):232-40.

[9]. D.B. Thordarson, T.P Hedman, D.N. Yetkinler, et al. Superior compressive strength of a calcaneal fracture construct augmented with remodelable cancellous bone cement. J Bone Joint SurgAm 1999;81(2):239-46.

[10]. T.A. Schildhauer, T.W. Bauer, C. Josten, et al. Open reduction and augmentation of internal fixation with an injectable skeletal cement for the treatment of complex calcaneal fractures. J Orthop Trauma 2001;14(5):309-17

[11]. G.S. Zhongguo. Sustentaculum tali screw fixation for the treatment of Sanders type II and III calcaneal fractures, 2015 Jan;28(1):315

[12]. G.S. Zhongguo, A study of 22 displaced intraarticular calcaneal fractures using locking plates with and without bone graft, 2011 Apr;24(4):3057.

[13]. V. Rak, D.Ira, M. Masek, Operative treatment of intra articular calcaneal fractures with calcaneal plates and its complications.Indian J orthop.July $2009 ; 43(3) 271-80$

[14]. C.F. Hyer, S. Atway, G.C. Berlet, T.H. Lee, Early weight bearing of calcaneal fractures fixated with locked plates .A radiographic review.Foot Ankle spec .Dec 2010;3(6);320-3 
[15]. H. Wang, Q. Zhang, D. Duan, et al. The use of calcaneal anatomic plate in arthroscopically-assisted open reduction and internal fixation of intra-articular calcaneal fractures.J Huazhong Univ Sci Technology Med Sci.2006;26(3):319-21

[16]. J.R. Stephenson. Surgical treatment of displaced intraarticular fractures of the calcaneus. Acombined lateral and medial approach.Clin Orthop Relat Res 1993;(290):68-75.

[17]. B.D. Burdeaux . Reduction of calcaneal fractures by the McReynolds medial approach technique and its experimental basis. Clin Orthop Relat Res 1983;(177):87-103.

[18]. B.D. Burdeaux. The medial approach for calcaneal fractures. Clin Orthop Relat Res 1993;(290):96-107.

[19]. I. Palmer. The mechanism and treatment of fractures of the calcaneus. J Bone Joint Surg. 1948;30A:2-8 